\title{
Incidental detection of accessory mitral valve in adolescent with Atrial Septal Defect: Diagnosis with 3D Transesophageal Echocardiography and Excision with Transaortic Method
}

\author{
Emine Azak ${ }^{1}$, Nuri Hakan Aydın ${ }^{1}$, Niyazi Ata Ecevit ${ }^{1}$, Ilker Çetin² ${ }^{2}$ and Zehra Golbasi ${ }^{2}$ \\ ${ }^{1}$ Ankara Sehir Hastanesi \\ ${ }^{2}$ Ankara Yildirim Beyazit Universitesi Tip Fakultesi
}

February 27, 2022

\begin{abstract}
Background: Accessory mitral valve tissue (AMVT) is an extremely rare causes left ventricular outflow tract (LVOT) obstruction and is usually incidentally detected in childhood. It is often associated with other cardiac and vascular congenital malformations. Case Presentation: In this case, we present a 15-year-old girl was diagnosed with AMVT by transesophageal echocardiography, resulting in LVOT obstruction during systole. Interestingly enough, the patient's accessory mitral valve remained undetected for years until he became symptomatic for wide ASD. Successful closure of the ASD with resection of the AMVT was performed with a transaortic approach. The patient was hemodynamically stable postoperatively. There were no abnormalities in the mitral valves and LVOT. Conclusion: It was also unusual to see AMVT with ASD instead of other frequently associated other congenital anomalies. Accessory mitral valve should be considered a rare but important cause of left ventricular outflow tract obstruction in childhood.
\end{abstract}

\section{INTRODUCTION}

Accessory mitral valve tissue (AMVT) is a rare cardiac anomaly in developing endocardial cushions. Frequently,it is associated with either simple or complex congenital heart disease, and rarely does it exist as an isolated lesion. Most symptoms occur when patients have a left ventricular outflow tract (LVOT) gradient of [?]50 mm Hg. Therefore, it is rare to discover an asymptomatic patient without LVOT obstruction. Accessory mitral valve tissue is usually diagnosed incidentally with complaints of chest pain, palpitations, dyspnea, and syncope in childhood. ${ }^{1-6}$ Surgical excision of isolated AMVT is required when there is significant obstruction of the LVOT and is associated with other cardiac abnormalities. Echocardiography provides important information about the morphology and hemodynamic significance of AMVT. ${ }^{6-8}$

Here, we present a patient with atrial septal defect (ASD) who was incidentally found to have AMVT on echocardiogram. Interestingly, the patient's accessory mitral valve remained undetected for years until he became symptomatic.

\section{CASE PRESENTATION}

A 15-year-old girl was referred to our hospital with a history of chest pain, shortness of breath, and presyncope. She had been suffering from chest pain since the age of 12 and had a history of frequent dizziness. The chest pain was atypical and had some non-specific features. She had no medical history and no family history of heart disease. On physical examination, her blood pressure was normal $(110 / 60 \mathrm{mmHg})$ and cardiac auscultation revealed a grade $2 / 6$ systolic murmur at the left sternal border. Laboratory tests revealed 
normal blood count, renal and liver function tests. Her ECG demonstrated right axis deviation, incomplete right bundle branch block and right ventricular dilation.

Two-dimensional transthoracic echocardiography (2D-TTE) revealed normal left ventricular size, mild left ventricular hypertrophy and systolic function. Left ventricular outflow tract showed a large, mobile, filamentous abnormal structure. The chordae of the anterior mitral leaflet were intact. This tissue was found to prolapse into the LVOT during systole. The morphology was suggestive of an AMVT. In addition, TTE and TEE showed a wide secundum-type ASD with moderate dilatation of the right heart chambers and mild aortic regurgitation (AR) (Fig 1).

However, the 2D-TTE images were not perfect and therefore three-dimensional transesophageal echocardiography (3D-TEE) was planned. Three-dimensional TEE provided anatomical information about other cardiac structures. Transesophageal echocardiography showed that the AMVT was attached to the ventricular portion of the anterior mitral valve leaflet, which prolapsed in and out of the LVOT, causing only mild LVOT obstruction (mean gradient $42 \mathrm{mmHg}$ ) and mild AR (Fig 2).

In this case, the physicians opted for surgical excision of the AMVT because of the development of LVOT obstruction and AR. In addition, the patient was not suitable for ASD transcatheter closure because of the flail atrial septum and insufficient aortic rim. Excision of the AMVT was easily achieved by aortotomy without the need for a left atriotomy. The ostium secundum ASD was closed with a pericardial patch. In the postoperative period, there was no LVOT obstruction and AR.

\section{DISCUSSION}

In some cases, AVMT is a rare anomaly in childhood and may be detected as an incidental finding or cause LVOT obstruction. This malformation is associated with other congenital heart malformations, such as atrial septal defect, ventricular septal defect, transposition of the large arteries, patent ductus arteriosus, aortic coarctation, bicuspid aortic valve, mitral cleft, and dextrocardia. ${ }^{7-9}$ The embryological mechanism of AMVT formation is not clear and may be related to abnormal or incomplete separation of the mitral valve from the endocardial cushions..$^{9-11}$ In the anatomical classification, there are three types of AVMT: type I: AMVT with attachments at the supra leaflet level, type II: AMVT attachments on the mitral leaflets and type III: AMVT attachments below the mitral leaflets.

Most cases of AMVT are associated with other cardiac malformations. The clinical signs and symptoms of this anomaly vary depending on its location and concomitant cardiac malformations. As in this case, congenital AMVT may remain asymptomatic for many years. In adult patients, AMVT may be diagnosed incidentally intraoperatively. Although AMVT can remain asymptomatic in patients, it can lead to LVOT obstruction in some patients. As in our case, most adolescent and adult cases are patients with vague symptoms in whom an echocardiography was performed for another indication. The most common symptoms are chest pain, dyspnea and palpitations. In addition, a few cases have been found to be associated with transient ischemic attack (TIA) and AMVT. ${ }^{7,10-13}$ The most common complaints in our patient were shortness of breath and atypical chest pain. Her neurological examination was normal and no TIA was detected in her medical history. The diagnosis of AMVT should be considered especially in patients with an unexplained cerebrovascular accident with a heart murmur and tissue causing signs of LVOT obstruction in the subaortic area.

Accessory mitral valve tissue resection is recommended in symptomatic isolated AMVT patients with a significant LVOT gradient (mean gradient [?]25 $\mathrm{mmHg}$ ) or in patients who were scheduled for cardiac surgery for additional cardiac pathology. ${ }^{7,10}$ Diagnosis of AMVT may be difficult before or during surgery. The echogenicity of AMV tissue is similar to that of endocardial structures. Therefore, surgeons should know the clinical and pathological features of AMVT to avoid overlooking it during surgery. ${ }^{1,7-10}$

Echocardiography is considered the gold standard for imaging AMVT. Transthoracic echocardiography (TTE) is sufficient for diagnosis, but perioperative TEE is important both for confirming the diagnosis and completing the surgical procedure, and for assessing postoperative mitral valve functions and possible 
complications that may occur after excision of AMVT. ${ }^{1-6,8}$ In particular, three-dimensional (3D) TEE allows assessment of the location of the AMVT attachment and the presence of an accompanying mitral lesion. We performed a TTE and a 3D-TEE which showed that the AMVT attached to the anterior mitral leaflet, with a subaortic attachment, resulting in an increase in the LVOT gradient.

Yasui et al. reported a patient who could not be separated from cardiopulmonary bypass due to LVOT caused by an overlooked AMVT, and pointed out that detection of this anomaly during surgery is difficult without an accurate presurgical diagnosis. ${ }^{14}$ Surgical excision of the AMVT should be carefully performed without affecting the functions of the mitral valve and surrounding structures. ${ }^{8,9,14}$

\section{CONCLUSIONS}

In conclusion, AMVT is an important cardiac lesion that is usually discovered incidentally. This case was presented because of its rarity in childhood. We emphasize that AMVT should be kept in mind in the differential diagnosis of LVOT obstruction, especially in adult patients with chest pain, dyspnea, and systolic murmur. A comprehensive evaluation is required to determine the need for surgical intervention. Recently, 3D echocardiography has played a fundamental role in patients' diagnosis, treatment, and followup. Congenital heart surgeons should be aware of the clinical and pathological features of AMVT to avoid overlooking them during surgery.

\section{Conflicts of Interest}

None.

\section{Disclosure}

Emine Azak and Nuri Hakan Aydin contributed equally to this work and should be considered co-authors. Niyazi Ata Ecevit, Ibrahim Ilker Cetin and Zehra Golbasi contributed equally to this work and should be considered co-corresponding authors.

\section{Declaration of Patient Consent}

The authors acknowledge that they have obtained written informed consent from the parents to present and publish this case report, including the images and associated text.

\section{Data Availability Statement}

The data that support the findings of this study are available from he corresponding author upon reasonable request

\section{REFERENCES}

1. Assaqqat M, Galal MO, G Baslaim, et al: Accessory mitral valve tissue in combination with subaortic membrane and significant impairment of left ventricular function. Pediatr Cardiol 2002: 23(1):87-9. doi: 10.1007/s00246-001-5023-7. Epub 2002 Feb 19.

2. Buttar R, Baibhav B: Non-Obstructive Accessory Mitral Valve Tissue in an Asymptomatic Adult: A Case Report and Review of Literature. Cureus 2020: 9;12(9):e10340. doi: 10.7759/cureus.10340.

3. Prifti E, Bonacchi M, Bartolozzi F, et al: Postoperative outcome in patients with accessory mitral valve tissue. Med Sci Monit 2003:9:126-133.

4. Popescu BA, Ghiorghiu I, Apetrei E, et al: Subaortic stenosis produced by an accessory mitral valve: the role of echocardiography. Echocardiography 2005:22(1):39-41. doi:10.1111/j.0742-2822.2005.03180.x.

5. Biyikoğlu SF, Güray Y, Ozturk S, et al: Asymptomatic accessory mitral valve tissue diagnosed by echocardiography. Anatol J Cardiol 2008: 8(3):5004-5005

6. Schmid AC, Zund G, Vogt P, et al: Congenital subaortic stenosis by accessory mitral valve tissue, recognition and management. Eur J Cardiothorac Surg 1999:15(4):542-544. doi:10.1016/s1010-7940(99)00023-8. 
7. Yuan SM, Shinfeld A, Mishaly D, et al: Accessory mitral valve tissue: a case report and an updated review of literature. J Card Surg 2008:23:769-772. doi: 10.1111/j.1540-8191.2008.00633.x.

8. Rovner A, Thanigaraj S, Perez JE, et al: Accessory mitral valve in an adult population: the role of echocardiography in diagnosis and management. J Am Soc Echocardiogr 2005:18:494-498. doi: 10.1016/j.echo.2005.01.027.

9. Yetkin E, Cuglan B, Turhan H, et al: Accessory mitral valve tissue: anatomical and clinical perspectives. Cardiovasc Pathol 2021:50:107277. doi: 10.1016/j.carpath.2020.107277. Epub 2020 Aug 31.

10. Manganaro R, Zito C, Khandheria BK, et al: Accessory mitral valve tissue: an updated review of the literature. Eur Heart J Cardiovasc Img 2014:15:489-497. doi: 10.1093/ehjci/jet163.

11. Al-Atta A, Khan H, Sosin M, et al: Accessory mitral valve tissue causing features of left ventricular outflow tract obstruction: a case report and updated literature review. J Ayub Med Coll Abbottabad 2019 $31: 276-278$.

12. Rao CM, Lucà F, Benedetto AF, et al: Accessory mitral valve: a rare cause of asymptomatic severe left ventricular outflow tract obstruction Ann Thorac Cardiovasc Surg. 2014:20:742-745. doi: 10.5761/atcs.cr.1300109. Epub 2013 Nov 8.

13. Okafor J, Kanaganayagam GS, Patel K, et al: A rare finding of giant accessory mitral valve tissue: a case report. Eur Heart J Case Rep 2020: 4:1-4. doi: 10.1093/ehjcr/ytz244.

14. Yasui H, Kado H, Kanegae Y,et al: Transventricular septal defect approach for resection of accessory mitral valve tissue. Ann Thorac Surg 1993:55:950-953. doi: 10.1016/0003-4975(93)90123-y.

\section{FIGURE LEGENDS}

Figure 1 (A) Two dimensional TTE image of the AVMT tissue attached to the ventricular side of the anterior mitral leaflet; (B,C) Two dimensional TEE image of the AMVT; (D) Two dimensional TEE image of the secundum ASD. Ao: Ascending aorta; LA: Left atrium; MV: Mitral valve, LV: Left ventricle; LVOT: Left ventricular outflow tract; AMVL: Accessory Mitral Valve Leaflet

Figure 2 (A,B) Three-dimensional TEE image of the AMVT; (C) Macroscopic image of the resected a AMVT
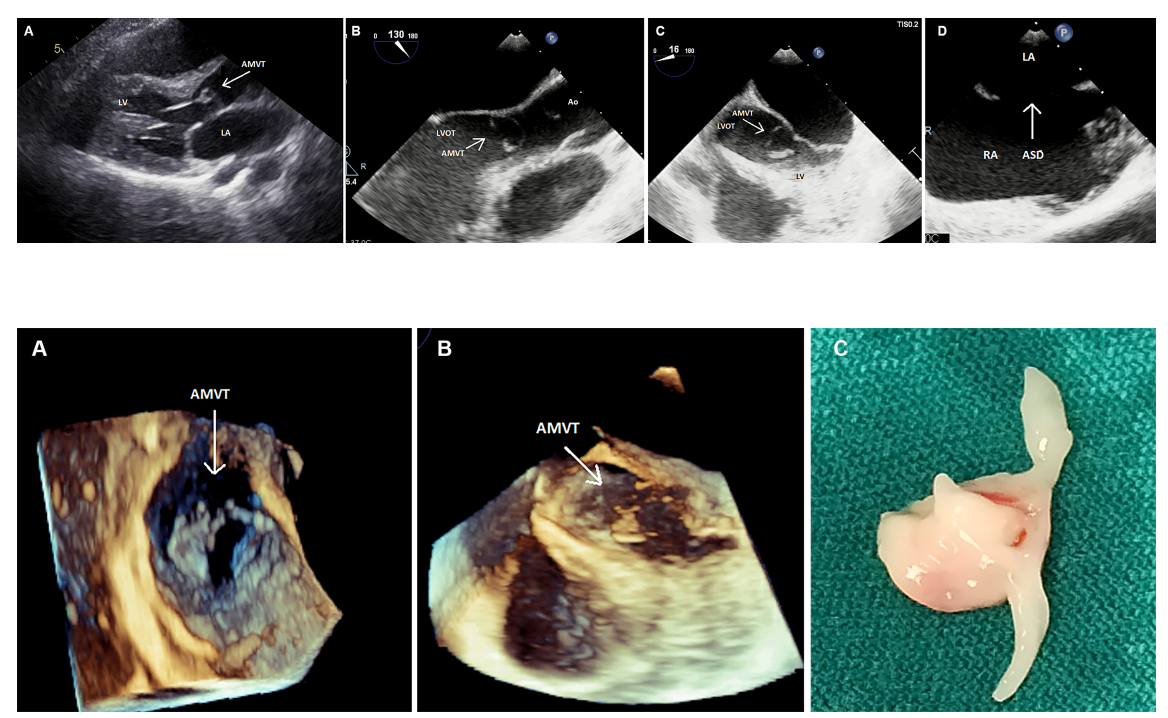